\title{
Increasing access to CBT for psychosis patients: a feasibility, randomized controlled trial evaluating brief, targeted CBT for distressing voices delivered by Assistant Psychologists (GiVE2)
}

Mark Hayward ( $\square$ mih21@sussex.ac.uk)

University of Sussex https://orcid.org/0000-0001-6567-7723

Clio Berry

University of Sussex

Ben Cameron

Pennine Care NHS Foundation Trust

Kate Arnold

Sussex Partnership NHS Foundation Trust

Katherine Berry

The University of Manchester

Stephen Bremner

Brighton and Sussex Medical School

Kate Cavanagh

University of Sussex

David Fowler

University of Sussex

Heather Gage

University of Surrey

Kathryn Greenwood

University of Sussex

Cassie Hazell

University of Westminster

Anna-Marie Jones

Sussex Partnership NHS Foundation Trust

Sam Robertson

Sussex Partnership NHS Foundation Trust

Clara Strauss

University of Sussex 


\section{Study protocol}

Keywords: Self-help, voice hearing, psychosis, CBT, supportive counselling

Posted Date: January 24th, 2020

DOl: https://doi.org/10.21203/rs.2.21826/v1

License: (c) (i) This work is licensed under a Creative Commons Attribution 4.0 International License. Read Full License

Version of Record: A version of this preprint was published at Trials on April 1st, 2020. See the published version at https://doi.org/10.1186/s13063-020-4181-y. 


\section{Abstract}

\section{Background}

The National Institute for Health and Care Excellence (NICE) recommends that Cognitive Behaviour Therapy for psychosis (CBTp) is offered to all patients with a psychosis diagnosis. However, only a minority of psychosis patients in England and Wales are offered CBTp. This is attributable, in part, to the resource-intensive nature of CBTp. One response to this problem has been the development of CBTp in brief formats that are targeted at a single symptom and the mechanisms that maintain distress. We have developed a brief form of CBTp for distressing voices and reported preliminary evidence for its effectiveness when delivered by highly trained therapists (Clinical Psychologists). This study will investigate the delivery of this intervention by a cost-effective workforce of Assistant Psychologists following a brief training and evaluate the acceptability and feasibility of conducting a future, definitive, randomised control trial (RCT).

\section{Methods}

This is a feasibility study for a pragmatic three-arm parallel group superiority 1:1:1 RCT comparing a Guided self-help CBT intervention for voices and treatment as usual (GiVE) to Supportive Counselling and treatment as usual (SC) to Treatment as usual alone (TAU), recruiting across two sites, with blinded posttreatment and follow-up assessments. A process evaluation will quantitatively and qualitatively explore stakeholder experience.

\section{Discussion}

Expected outcomes will include an assessment of the feasibility of conducting a definitive RCT and data to inform the calculation of its sample size. If evidence from a subsequent fully powered RCT suggests that GiVE is clinically and cost effective when delivered by briefly trained Assistant Psychologists, CBTp offered in these less resource-intensive forms has the potential to generate benefits for individual patients (reduced distress, enhanced recovery and enhanced quality of life), service-level patient benefit (increased access to evidence-based psychological therapies) and economic benefits to the NHS (in terms of the reduced use of mental health inpatient services).

\section{Administrative Information}


Title

Increasing access to CBT for psychosis patients: a feasibility, randomized controlled trial evaluating brief, targeted CBT for distressing voices delivered by Assistant Psychologists (GiVE2)

Trial registration .

Current Controlled Trials ISRCTN registration number: 16166070. Registered on 05 February 2019. http://www.isrctn.com/ISRCTN16166070

Protocol version

Version 1 , dated $05 / 11 / 18$

Funding

This project is funded by the National Institute for Health Research for Patient Benefit (project number PB-PG-0317-20029). The views expressed in this publication are those of the authors and not necessarily those of the NHS, $\mathrm{NIHR}$ or the Department of Health. 
Title

Increasing access to CBT for psychosis patients: a feasibility, randomized controlled trial evaluating brief, targeted CBT for distressing voices delivered by Assistant Psychologists (GiVE2)

Author details

Mark Hayward (corresponding author)

School of Psychology, University of Sussex, Brighton BN1 9RH \& Research \& Development Department, Sussex Partnership NHS Foundation Trust, Hove, BN3 7HZ

m.i.hayward@sussex.ac.uk

Clio Berry

School of Psychology, University of Sussex, Brighton BN1 9RH

c.berry@sussex.ac.uk

Ben Cameron

Research \& Development Department, Pennine Care NHS Foundation Trust, 225 Old St, Ashton-under-Lyne OL6 7SR

Ben.cameron@nhs.net

Kate Arnold

Research \& Development Department, Sussex Partnership NHS Foundation

Trust, Hove, BN3 7HZ

Kate.arnold2@sussexpartnership.nhs.uk

Katherine Berry

Faculty of Biology, Medicine \& Health, University of Manchester, M13 9PL

Katherine.berry@manchester.ac.uk

Stephen Bremner

Brighton \& Sussex Medical School, University of Sussex, Brighton BN1 9RH

s.bremner@sussex.ac.uk

Kate Cavanagh

School of Psychology, University of Sussex, Brighton BN1 9RH

k.cavanagh@sussex.ac.uk

David Fowler

School of Psychology, University of Sussex, Brighton BN1 9RH

D.Fowler@sussex.ac.uk

Heather Gage

School of Biosciences \& Medicine, University of Surrey GU27XH

h.gage@surrey.ac.uk

Kathryn Greenwood

School of Psychology, University of Sussex, Brighton BN1 9RH

k.e.greenwood@sussex.ac.uk

Cassie Hazell

Social Sciences Department, University of Westminster, London W1W 6UW

c.hazell@westminster.ac.uk

Anna-Marie Jones

Research \& Development Department, Sussex Partnership NHS Foundation

Trust, Hove, BN3 7HZ

Anna-marie.jones@sussexpartnership.nhs.uk

Sam Robertson

Research \& Development Department, Sussex Partnership NHS Foundation

Trust, Hove, BN3 7HZ

Sam.robertson@sussexpartnership.nhs.uk

Clara Strauss

University of Sussex \& Sussex Partnership NHS Foundation Trust, BN3 7HZ c.y.strauss@sussex.ac.uk

Name and contact information for the trial sponsor
Sussex Partnership NHS Foundation Trust, Millview, Nevill Avenue, Hove BN3 7HZ - researchgovernance@sussexpartnership.nhs.uk 
Title

Increasing access to CBT for psychosis patients: a feasibility, randomized controlled trial evaluating brief, targeted CBT for distressing voices delivered by Assistant Psychologists (GiVE2)

Role of sponsor

The study is sponsored by Sussex Partnership NHS Foundation Trust who will provide at least annual monitoring and audit of the trial and governance procedures.

\section{Introduction}

\section{Background and rationale}

Cognitive Behaviour Therapy for psychosis (CBTp) has robust evidence for clinical ${ }^{1,2,3}$ and cost effectiveness ${ }^{4}$ and is recommended by the National Institute for Health and Care Excellence (NICE) to promote the recovery of psychosis patients. ${ }^{4}$ However, implementation in the UK is extremely poor, ${ }^{5}$ with only $26 \%$ of psychosis patients being offered CBTp. ${ }^{6}$ The most consistently reported barrier to implementation is insufficient resources, including a lack of trained therapists with dedicated time to deliver CBTp. ${ }^{7}$

The Department of Health's Forward View for Mental Health ${ }^{8}$ and Long Term Plan ${ }^{9}$ include a commitment to increase access to evidence-based psychological therapies for patients with psychosis. However, future investments in additional trained therapists with dedicated time to deliver CBTp are unlikely to be made on a large enough scale to significantly and comprehensively increase access to CBTp in the near future. Consequently, additional strategies for increasing access may be required, including a reduction in the resources required to deliver CBTp.

$\mathrm{NICE}^{4}$ recommend research into two issues that could potentially reduce the resources required to deliver CBTp. Firstly, the duration of CBTp - how many sessions are required to generate sufficient benefit? Our meta-analysis of 10 controlled studies indicated that CBTp delivered over less than the recommended 16 sessions is effective in reducing psychosis symptoms when delivered by trained therapists (typically Clinical Psychologists). ${ }^{10}$ This focus upon shorter forms of CBTp has recently been taken forward through the development of single-symptom therapies ${ }^{11}$ which target only one specific psychotic symptom, with some promising early results. ${ }^{12,13,14,15}$ Secondly, the ability of "briefly trained" practitioners to deliver CBTp - can CBTp be delivered by a more cost effective alternative workforce (i.e. not highly trained therapists)? Evidence to date suggests that case managers cannot fulfil this role as outcomes from trials have been inconclusive $e^{16,17,18,19}$ and there are competing demands upon their time. ${ }^{18,19}$ Psychology graduates, however, are well positioned to undertake such a role: ${ }^{18,20}$ there are a large number of psychology graduates in the UK, US and elsewhere; their degree provides them with training in psychological models of emotion and behaviour; and they can be readily employed in Assistant Psychologist (AP) roles at lower cost than more specialist therapists. 
Our response to the questions posed by NICE was to develop a brief form of CBTp targeted at distressing voices. We collaborated with Lived Experience Consultants to turn our self-help CBT book (Overcoming Distressing Voices ${ }^{21}$ ) into a Guided self-help CBT intervention package (GiVE) supported by a workbook. Our first step was to demonstrate the validity and viability of the GiVE intervention package, confirming it can be delivered by expert therapists with benefits for patients. We conducted a pilot RCT of GiVE compared to usual care $(\mathrm{N}=28) .{ }^{22}$ Results were encouraging with high levels of retention within the study $(96 \%)$, and a very large between-groups effect for the pre-determined primary outcome of negative voice impact $(d=1.78) .{ }^{23}$

Our second step within this current study will be to assess the feasibility of conducting an RCT to evaluate the outcomes of GiVE when it is delivered by briefly trained APs. If found to be feasible, the definitive RCT will form the third step within our programme of research. If GiVE is found to be clinically and cost effective in a future definitive RCT when delivered by APs, it could substantially increase the number of psychosis patients that are able to access CBTp, which will generate benefits for psychosis patients and the NHS.

\section{Objectives}

The long-term aim of this programme of research is to increase access to CBTp for psychosis patients. This aim will be achieved if a brief and targeted CBTp intervention delivered by a less costly and workforce of briefly trained APs is found to be clinically and cost effective.

The specific aim of the current study is to explore the feasibility of conducting a three-arm RCT of GiVE delivered by APs. A process evaluation, using mixed methods and drawing on Normalization Process Theory, ${ }^{24}$ will be used to explore mechanisms and contextual factors that may affect the future uptake and implementation of the intervention, in both research and clinical contexts.

Specifically, this feasibility study aims to assess: 1) the acceptability of the brief and targeted intervention to clinicians (will they refer patients to the study?);2) the acceptability of the brief and targeted intervention to patients (can patients be recruited and retained, and what are their experiences of the intervention?); and 3) the ability of APs to adhere to the therapy and clinical supervision protocols. We will also estimate the standard deviation of outcomes in order to facilitate a sample size calculation for use within a definitive trial.

\section{Trial Design}

This is a feasibility RCT with a three-arm parallel group superiority 1:1:1 allocation, comparing a Guided self-help CBT intervention for VoicEs and treatment as usual (GiVE) to Supportive Counselling and treatment as usual (SC) to Treatment as Usual alone (TAU), recruiting across two sites, with blinded posttreatment and follow-up assessments. Outcomes will be assessed at baseline (pre-randomization - Time 
0), 16 weeks (post-intervention - Time 1), and 28 weeks (follow-up - Time 2). Time 1 and 2 assessments will be conducted blind by a researcher who is blind to group allocation. Adherence to the therapy protocol will be assessed by APs after each session. AP competence will be assessed by independent raters reviewing a random selection of early, middle, and late recorded sessions.

A mixed methods process evaluation drawing upon Normalization Process Theory ${ }^{24}$ will be used to capture participants', clinicians' and APs' experiences of the study and the interventions (GiVE and SC), and their views on the facilitators and barriers to the implementation of GiVE within routine psychosis care pathways. The results will be used to develop logic models for a future trial and for NHS implementation.

\section{Methods: Participants, Interventions And Outcomes Study setting}

Participants will be recruited from two sites within the UK National Health Service (NHS) - Sussex Partnership NHS Foundation Trust and Pennine Care NHS Foundation Trust.

\section{Eligibility criteria}

Inclusion criteria:

1. Aged 16 years or older;

2. In contact with Secondary Care Mental Health Services (under the care of a Consultant Psychiatrist);

3. Experiencing current voice hearing; this will be operationalized by participants having a score of at least 1 on item 1 (How frequently did you hear a voice or voices?) on the Hamilton Program for Schizophrenic Voices Questionnaire (HPSVQ) ${ }^{25}$ - indicating that the participant has experienced at least one episode of voice hearing in the past week;

4. Distressed by hearing voices; operationalized by participants scoring at least 8 out of 16 on the 'negative impact' scale of the HPSVQ 25 ;

5. Meeting DSM-5 Research criteria for Schizophrenia Spectrum or Other Psychotic Disorders (assessed by the Structured Clinical Interview for DSM-5 disorders, SCID- $5^{26}$ );

6. Willing and able to provide written, informed consent.

Exclusion criteria:

1. Established organic cause for distressing voices (e.g. brain disease or injury);

2. Primary diagnosis of substance misuse;

3. Currently detained in hospital under a section of the Mental Health Act;

4. Have completed a full course (minimum of 16 hours) of CBT for psychotic symptoms during the past year; 
5. Be currently participating, or be confirmed to participate in another interventional study in which they are receiving an intervention which utilizes psychological therapy;

6. Non-English speaking to the degree that the participant is unable to fully understand and answer assessment questions or give informed consent;

7. Severe learning disability - assessed using the Test of Premorbid Functioning - UK (TOPF-UK). ${ }^{27}$

8. Immediate and serious risk to self or other (assessed at the point of referral/eligibility review).

\section{Who will take informed consent?}

Once a formal referral has been received by the research team, the potential participant will be contacted to discuss the study further and arrange a consent and eligibility meeting with a member of the research team. The potential participant will have a copy of the PIS at least 24 hours before the consent and eligibility meeting takes place, so they will have time to read the information, discuss it with friends and family, and formulate any questions they may have.

\section{Blinding}

The RAs and the Trial Statistician will be blind to the allocation sequence. Following the baseline assessment, participants will be randomised using Sealed Envelope and allocated to one of the three trial arms. Only the Trial Manager will receive this notification and will communicate the allocation to all unblinded members of the research team. Researchers will be blinded to the allocation and will remain blinded for all future assessments with the participants ( 16 week and 28 week data collection). Measures to maintain blinding will include: 1) participants being reminded at the beginning of each assessment interview to not disclose the group to which they have been allocated; 2) blinded members of the research team being shielded from discussion of participants in forums where the possibility of determining participant allocation could occur; 3 ) researcher access to electronic health records being restricted; and 4) consideration given to office allocation and all administrative processes of blinded-vs-unblinded members of the research team. 'Blind' awareness and education will be promoted throughout the study. To test the success of blinding, the blind RA who assesses each participant will be asked to guess the allocation group for each participant at the end of the final assessment.

Reported breaks in blinding will be recorded. Outcome assessments will be re-blinded by re-allocating 'blind' RAs to collect and score study data wherever possible.

\section{Interventions}

\section{Explanation for the choice of comparators}

A three-arm RCT was chosen to enable: 1) any effect of GiVE to be differentiated from two other components of the response to talking therapies (the concerned attention and generic therapeutic effects 
- both available within SC); and 2) an evaluation of the cost effectiveness of GiVE - generated by a comparison to the treatments that are usually offered to patients (available within TAU).

\title{
Intervention description
}

\author{
Guided self-help intervention for voices (GiVE)
}

The GiVE intervention will be delivered by an AP and will follow a workbook ${ }^{28}$ that is based upon the Overcoming Distressing Voices ${ }^{21}$ self-help book. Participants will be given a copy of both the workbook and the self-help book at the commencement of therapy by the AP and asked to engage in some level of self-help (homework); this will take the form of reading chapters from the self-help book between therapy sessions and engaging with the suggested activities within the workbook. Participants in the GiVE intervention will also have the opportunity to access the 'CHOICES' mobile phone application.

After an introductory session on coping, the intervention will cover three core modules: (1) beliefs about the self, (2) beliefs about voices, and (3) relationships. Modules (1) and (2) draw upon psychoeducation and cognitive behavioural strategies to help participants to re-evaluate their negative or unhelpful beliefs related to the self and voices. Module (3) additionally involves work on how to relate to others and voices more assertively. GiVE will be delivered over eight sessions offered over a maximum of 16 weeks. Sessions may be delivered in NHS clinics, participants' own homes and other community locations as preferred and appropriate. Each session will last up to one hour, and sessions will be held weekly where possible ( 16 weeks are given for the 8 sessions to allow for periods of absence, illness, holidays, etc.). Participants in the GiVE arm will continue to receive their usual treatment throughout their participation in the study.

\section{Supportive Counselling}

Supportive Counselling (SC) will follow the therapy protocol that was used by APs in the full RCT of AVATAR Therapy for distressing voices. ${ }^{29}$ It will be delivered over the same number and duration of sessions as GiVE, with the aim of matching the duration of total therapist contact time across both arms. SC will offer a supportive and non-judgemental space for the discussion of topics and issues determined by the participant. The SC therapy protocol contains specific guidance for the AP on how to respond to participant disclosure of distressing voices in a manner that does not provide specific intervention strategies.

The intervention will involve 8 sessions over a maximum of 16 weeks. Each session will last up to one hour, and sessions will be held weekly where possible ( 16 weeks are given for the 8 sessions to allow for periods of absence, illness, holidays, etc.). Sessions may be delivered in NHS clinics, participants' own homes and other community locations as preferred and appropriate. Participants in the SC arm will continue to receive their usual treatment throughout their participation in the study.

The same APs will deliver GiVE and SC in order to minimize the influence of therapist effects, for example, differing levels of therapist competence. They will receive a 5-day training in GiVE and SC (one 
introductory day, two days on GiVE and two days on SC), delivered by experienced Clinical Psychologists and an experienced Counselling Psychologist, respectively. Weekly clinical supervision will be provided by these Clinical/Counselling Psychologists.

Treatment-as-usual

Treatment as usual (TAU) will be provided by the usual care team. TAU should be informed by NICE guidelines (NICE) ${ }^{4}$ and typically include medication management and support and monitoring provided by the Adult Mental Health or Early Intervention in Psychosis Services, with individual and family psychological therapies offered occasionally.

\section{Strategies to improve adherence to interventions}

Therapeutic drift and contamination will be minimized by the use of highly detailed therapy protocols and close supervision of the APs by experienced Clinical/Counselling Psychologists. Adherence to therapy protocols will be assessed through APs completing a checklist at the conclusion of each session. The competence of AP delivery of the interventions will be assessed by the rating of session recordings. All therapy sessions will be audio-recorded (with the participant's permission) and a random $10 \%$ sample rated for competence by independent experts (a Clinical Psychologist for GiVE and a Counselling Psychologist for SC). GiVE sessions will be rated using the Cognitive Therapy Rating Scale for Psychosis. ${ }^{30}$ SC sessions will be rated using the Counselling Adherence Scale. ${ }^{29}$

\section{Relevant concomitant care permitted or prohibited during the trial}

Participants in all three arms of the trial will be encouraged to engage in and continue with existing treatments. Our methodological approach will be to carefully monitor and capture the service contacts received across a range of services in all three arms of the trial using an adapted version of the Client Service Receipt Inventory (CSRI). ${ }^{31}$ Within a future definitive RCT, this will allow us to standardize current practice by providing all referrers with a best practice manual for standard treatment which summarises good practice.

\section{Outcomes}

The assessment of feasibility will include the calculation of the number and proportion of: CCs willing to refer their patients; referred patients found to be eligible; consenting participants who are retained within the study and offer full data sets; non-missing items for each variable; consenting participants within the GiVE and SC arms who reach the point of therapy 'exposure' (attended at least 6 of 8 therapy sessions). Feasibility assessment will also measure therapist adherence to therapy and supervision protocols.

\section{Participant timeline}

Figure 1 illustrates the trial/recruitment flowchart. 


\section{Sample size}

Following recommendations ${ }^{32}$ for designing feasibility trials that aim to detect a small-medium standardized effect size (where the definitive trial will be designed with $90 \%$ power and two-sided $5 \%$ significance), this study aims to recruit 90 patients. ${ }^{33,34}$ Participants will be in contact with Community Secondary Care Adult Mental Health Services or Early Intervention in Psychosis services at the time of consent, across two sites in the UK: (1) Sussex Partnership NHS Foundation Trust (suburban and rural); and (2) Pennine Care NHS Foundation Trust (suburban and urban).

\section{Recruitment}

Participants will be recruited through referrals from the Care Co-ordinators' (CCs) of psychosis patients (or other appropriate members of the clinical team) in the host sites. When first approached about the study by a Research Assistant (RA), all CCs will be given a participant information sheet (PIS) and referral forms. CCs will be asked to share the PIS with any patients who are potentially eligible for the study. If a patient shows an interest in the study and gives their verbal agreement to be contacted by a researcher, the $\mathrm{CC}$ will be asked to complete a referral form.

An additional recruitment strategy at the Sussex site will involve the use of the Everyone Counts scheme. Sussex Partnership NHS Foundation Trust has the Everyone Counts scheme in place for consent to contact about research opportunities. Members of the Research and Development Department will contact potentially eligible patients to discuss the study and invite them to take part. Interested patients will be able to contact either the Research and Development Department or the research team directly and enquire about the study. They will be invited to discuss the study with their $\mathrm{CC}$ who can then complete a referral form. The research team could support potential participants to discuss the study with their CC if this is needed. An additional strategy will be used in Pennine Care NHS Foundation Trust, whereby individuals who have participated in other trials within the Trust and have given consent to be contacted about future studies may be contacted.

\section{Assignment Of Interventions: Allocation}

\section{Sequence generation and Concealment mechanism}

Participants will be randomly allocated using the Sealed Envelope online service https://www.sealedenvelope.com/. The trial statistician will set up and test the randomization procedure incorporating stratification by site (Sussex or Pennine) and type of service (Community Mental Health Team or Early Intervention in Psychosis) using random block lengths and 1:1:1 allocation. Participants will be randomly allocated to receive either the study intervention and treatment as usual (GiVE) or the control intervention and treatment as usual (SC) or Treatment as Usual (TAU).

\section{Implementation}


Consented participants will be randomised by the Trial Manager using Sealed Envelope. An unblinded member of the research team will then contact the participant by telephone to inform them of their group allocation. APs will be notified by the Trial Manager about the participants who are allocated GiVE and SC and will be asked to arrange a first appointment, if possible, within two weeks following the randomisation. A letter will be sent to all participants to confirm their allocation and details of their next appointment with either the AP (GiVE or SC) or researcher (TAU). This letter will ask participants not to disclose their allocation to the researcher.

\section{Assignment Of Interventions: Blinding Who will be blinded?}

The RAs and the Trial Statistician will be blind to the allocation sequence. Following the baseline assessment, participants will be randomised using Sealed Envelope and allocated to one of the three trial arms. Only the Trial Manager will receive this notification and will communicate the allocation to all unblinded members of the research team. Researchers will be blinded to the allocation and will remain blinded for all future assessments with the participants (16 week and 28 week data collection). Measures to maintain blinding will include: 1) participants being reminded at the beginning of each assessment interview to not disclose the group to which they have been allocated; 2) blinded members of the research team being shielded from discussion of participants in forums where the possibility of determining participant allocation could occur; 3 ) researcher access to electronic health records being restricted; and 4) consideration given to office allocation and all administrative processes of blinded-vs-unblinded members of the research team. 'Blind' awareness and education will be promoted throughout the study. To test the success of blinding, the blind RA who assesses each participant will be asked to guess the allocation group for each participant at the end of the final assessment.

\section{Procedure for unblinding if needed}

Reported breaks in blinding will be recorded. Outcome assessments will be re-blinded by re-allocating 'blind' RAs to collect and score study data wherever possible.

\section{Data Collection And Management}

\section{Plans for assessment and collection of outcomes}

See Fig. 2 for details of assessment at each visit.

Screening Measures

The assessment of eligibility in relation to the inclusion and exclusion criteria will be supported by the use of the Structured Clinical Interview for DSM-5 Disorders (SCID-5), ${ }^{26}$ the Test of Premorbid Functioning - 
UK (TOPF-UK), ${ }^{27}$ and the Hamilton Program for Schizophrenia Voices Questionnaire (HPSVQ) ${ }^{25}$ (each described below).

Clinical Measures - Primary

The primary outcome for a potential future definitive RCT is the self-reported impact of voice-hearing upon the participants. Two measures will be evaluated as candidates for the primary outcome to be used in a future definitive RCT:

Psychotic Symptoms Rating Scale (PSYRATS-AH) ${ }^{35}$ Distress Scale - PSYRATS-AH is an 11 -item rating scale designed to measure the severity of different dimensions of the voice hearing experience. Items are grouped together in four factors ${ }^{36}$; distress (negative content, distress, and control), frequency (frequency, duration, and disruption), attribution (location and origin of voices), and loudness (loudness item only). The 'distress' subscale measures the impact that voices have on the individual. The measure has established psychometric properties.

Hamilton Program for Schizophrenia Voices Questionnaire (HPSVQ) ${ }^{25}$ Negative Impact Scale - HPSVQ is a 9-item self-report measure of the phenomenology and negative impact of voice hearing. The 'negative impact' subscale has four items rated on a 5-point (0-4) scale and measures the level of distress and impact that voices have on the person. HPSVQ has been found to have strong concurrent validity (all $r>0.80$ ) as well as good internal consistency (all $a>0.82$ ) in psychosis patients. ${ }^{37}$

Clinical Measures - Secondary

Secondary outcomes will evaluate: 1) mental health problems commonly experienced by people with psychosis (anxiety and depression [Hospital Anxiety and Depression Scale] ${ }^{38}$ and paranoia [Paranoid Thoughts Scale] ${ }^{39}$ ): 2) variables that have been associated with the impact of voice-hearing (negative beliefs about the self [Self Scale of the Brief Core Schema Scale], ${ }^{40}$ negative beliefs about voices [Beliefs About Voices Questionnaire - Revised], ${ }^{41}$ and negative relating to voices [Voice and You] ${ }^{42}$ and other people [Persons Relating to Others Questionnaire] ${ }^{43}$; and 3) a range of personal and social recoveryoriented outcomes (goals for the outcome of CBTp [CHOICE - short form], ${ }^{44}$ the positive impact of voices [Voice Impact Scale], ${ }^{45}$ engagement in meaningful activity [Work and Social Adjustment Scale] ${ }^{46}$ and social functioning [Social and Occupational Functioning Scale]. ${ }^{47}$

Process measures

Process measures will evaluate the quality of the therapeutic relationships (Scale to Assess Therapeutic Relationships) ${ }^{48}$ and participants' expectations for therapy (Therapy Credibility and Expectancy Questionnaire). ${ }^{49}$

Health economic measures 
EQ5D-5L 50 - is a standardized instrument used as a measure of health-related quality of life that can be used in a wide range of health conditions and treatments. The descriptive system comprises five dimensions: mobility, self-care, usual activities, pain/discomfort and anxiety/depression. Each dimension has five levels: no problems, slight problems, moderate problems, severe problems and extreme problems.

Client Service Receipt Inventory (CSRI-UK) ${ }^{31}$ - is a well validated adaptable research instrument used in mental health settings to collect information on health, social and voluntary service utilization, informal care, accommodation, other public services (e.g. police) and private out-of-pocket expenses incurred. Its primary purpose is to allow resource-use patterns to be described and costs to be estimated.

Short Form 12 (SF12) ${ }^{51}$ - this generic health survey captures information about functional health and well-being from the patient's point of view.

\section{Plans to promote participant retention and complete follow- up}

Efforts will be made to engage all participants in follow-up assessments. RAs will flexibly engage patients, offering appointments at times and locations which best suit participants and offering shorter and split assessment sessions as needed. Participants will be offered reimbursement of $£ 20$ per assessment point (i.e. baseline, 16 and 28 weeks) and travel expenses will be made available. Retention rates will be monitored by the Trial Manager at least weekly and the research team on a monthly basis throughout the trial. The LEAP will be asked to provide consultation regarding retention rates as part of their oversight role.

\section{Data management}

Data collection and analysis will be supervised by the Trial Statistician. The management of the data will be a standing item on the agenda of the monthly meeting of the research team. All members of the research team and any other individuals from collaborating Trusts or Universities involved in collecting, inputting, processing, using and sharing data will have had Information Governance training.

\section{Confidentiality}

The minimum amount of personal information needed to conduct the study will be obtained from participants. Personal and research data will be stored securely on NHS premises. Physical data, such as consent forms, will be locked in filing cabinets on NHS premises accessible only to members of the research team. Electronic data will be stored securely in password-protected or encrypted files on NHS computers accessible only to members of the research team. All research data will be fully anonymised and will be stored separately to personal data. A link file will allow for participant research data to be identified. This link file will be a password-protected file accessible only by members of the trial team within each research site and the Trial Manager. This file will be securely destroyed following the end of the study. Quantitative and qualitative data will be appropriately aggregated in any publications arising from the trial to protect participant anonymity during and after the trial has ended. 


\section{Statistical Methods}

\section{Statistical methods for primary and secondary outcomes}

Analyses will be based on the intention-to-treat approach. All clinical outcomes will be summarised using descriptive statistics at pre-randomisation (T0), at 16 weeks (T1) and at 28 weeks (T2) for each arm (GiVE, SC \& TAU) of the study. Next, $95 \%$ \& 75\% confidence intervals ${ }^{51}$ will be created for between group mean differences after adjustment for the baseline scores using ANCOVA for the following comparisons: GiVE vs TAU; GiVE vs SC; and TAU vs SC. There will be no hypothesis testing.

Analysis will be conducted using a linear mixed model with a random effect for individual with fixed effects for treatment group (TAU, GiVE and SC) and time (16 and 28 weeks); baseline score and site will be entered as covariates. Contrasts will be used to compare groups (GiVE vs TAU, GiVE vs SC \& TAU vs SC) at T1 and T2. 95\% \& 75\% confidence intervals ${ }^{25}$ for between-group mean differences will be created for all estimates of unstandardised effect size.

Standardised (Cohen's d) effect sizes for each outcome will be calculated by dividing the between-group unstandardized effect by the baseline pooled standard deviation. The level of missing data will be summarised for each outcome and reported for each time point.

Two potential primary outcomes - Distress scale of PSYRATS and the Negative Impact scale of the HPSVQ - are being explored in this feasibility study. In addition to the analyses for all clinical outcomes, the between-groups differences for these two variables will be assessed in the context of:

- Whether the Minimal Clinically Important Difference (MCID) is contained within the $95 \%$ Confidence Intervals where:

- PSYRATS Distress scale of MCID is a 3-point reduction

- HPSVQ Negative Impact scale MCID is a 2-point reduction

- The level of missing data

- Popularity of the outcome in the literature

- Any other relevant information generated by the process evaluation.

\section{Methods for additional analyses (e.g. subgroup analyses)}

Health Economic analysis

An economic evaluation will provide basic information to facilitate the planning of a definitive, multicentre RCT to assess clinical and cost effectiveness. The resource requirements and costs of each arm will be investigated using trial details on the delivery of GIVE and SC. Staff time will be costed using national tariffs, inclusive of on-costs and overheads. ${ }^{52}$ Participants will be asked to complete the CSRI-UK at 16 weeks (end of intervention) and 28 weeks to record all forms of service use (including activity 
classified as usual care), informal care and out-of-pocket expenses. Information gathered through the CSRI-UK will be cross checked for accuracy with mental health records. Discrepancies between these records will be recorded and evaluated. Health-related quality of life (HRQoL) will be recorded at baseline and 16 and 28 weeks using both EQ-5D-5L and SF-12 for calculation of QALYs as the latter may be more sensitive to changes in the psychological status. Completeness of data will be assessed. Variability in standard care will be explored, and the use of EQ-5D-5L and SF12 compared. Differences in service use between groups will be investigated for indications of potential savings associated with GiVE that might offset the intervention costs. The full range of outcomes will be investigated in a cost consequences framework and a preliminary cost-effectiveness analysis conducted.

Adherence and Competence Analysis

Therapeutic drift and contamination will be minimized by the use of highly detailed therapy protocols and close supervision of the APs by experienced Clinical/Counselling Psychologists. Adherence to therapy protocols will be assessed through APs completing a checklist at the conclusion of each session. The competence of AP delivery of the interventions will be assessed by the rating of session recordings. All therapy sessions will be audio-recorded (with the participant's permission) and a random $10 \%$ sample rated for competence by independent experts (a Clinical Psychologist for GiVE and a Counselling Psychologist for SC). GiVE sessions will be rated using the Cognitive Therapy Rating Scale for Psychosis. ${ }^{30} \mathrm{SC}$ sessions will be rated using the Counselling Adherence Scale. ${ }^{29}$

Process Evaluation

The process evaluation will be guided by MRC Guidelines ${ }^{53}$ and used to evaluate:

1. How the intervention was delivered - qualitative interviews with the APs delivering GiVE regarding their experience of the training, manuals, supervision and intervention delivery;

2. Implementation of the intervention - what was delivered in terms of exposure (number of sessions offered and received by participants) and therapist adherence to protocol;

3. Reach of the intervention - the proportion of eligible participants who are referred and who accept the offer of therapy, responses from referring and non-referring clinicians to a questionnaire designed to explore their decision-making around referring patients with psychosis to CBT generally and the GiVE intervention specifically;

4. Context - qualitative interviews with clinicians and managers from referring services, to determine the potential actions, context (team/service/supervisor issues), processes, structures and coherence with standard care which might influence implementation of the GiVE intervention, including sense making, and potential effort, action, commitment, participation and reflection on implementation of GiVE in routine services;

5. Participant experience - of the research process, randomization, outcomes, potential mechanisms and mediators, and therapy. 
Descriptive data will be used to summarise reach and implementation of the intervention. Qualitative data from the process evaluation interviews will be analysed using thematic analysis ${ }^{54}$; this method will be used to highlight the key themes across interviews in relation to the research questions. These themes will be used to adapt the study design, manuals, training, supervision and intervention delivery where necessary before proceeding to a definitive trial. In addition, the qualitative data will be used to develop both: (1) a system-based logic model for the implementation of the trial and subsequent therapy; and (2) a process-based logic model of the potential mechanisms from therapy experience to outcomes. These will be further refined in the future definitive RCT.

\section{Oversight And Monitoring}

\section{Composition of the trial steering committee}

Medical Research Council Guidelines on Good Clinical Practice in Clinical Trials ${ }^{55}$ informed the constitution of the Trial Steering Committee (TSC), which includes an independent chair, independent experts and lay members.

The scientific integrity of the trial will be overseen by the TSC. The TSC will also serve the functions of a Data Monitoring and Ethics Committee (DMEC), as a combined TSC and DMEC is appropriate within the size and scope of the present feasibility trial, including oversight of the safety and data integrity of the trial.

A separate Lived Experience Advisory Panel (LEAP) will provide Patient and Public Involvement (PPI) oversight of the trial. The LEAP will consult at regular intervals throughout the trial (approximately six meetings in total) and will advise on issues relating to design, delivery, interpretation of findings, dissemination and preparation for the planned definitive RCT. Prior to the submission of the grant application for current study, the LEAP reviewed the differences between the training and role of Clinical Psychologists (who delivered GiVE in our initial Pilot RCT) and APs (who will deliver GiVE in the current study) and emphasized the need for APs to have experience of working with people with psychosis. This became an essential criterion for appointment to the AP post.

\section{Adverse event reporting and harms}

Any unfavourable and unintended sign, symptom or illness that develops or worsens during the period of the study will be classified as an adverse event $(\mathrm{AE})$, whether or not it is considered to be related to the study treatment. Adverse events will include: an exacerbation of a pre-existing illness; an increase in the frequency or intensity of a pre-existing episodic event or condition; a condition that is detected after trial intervention administration; and continuous persistent disease or a symptom present at baseline that worsens following administration of the trial treatment - and may be expected or unexpected. Serious Adverse Events (SAEs) are those considered to be life-threatening, resulting in death, requiring inpatient hospitalisation or prolongation of existing hospitalisation, resulting in significant or persistent 
incapacity/disability or a birth defect or congenital abnormality. The number (events and individuals) and nature of all events ( $A E$ and $S A E$ ) reported to blind and unblind members of the research team will be recorded.

The period for adverse event reporting will be following the signing of the study consent form until last follow-up assessment 28 weeks after randomisation. All AEs will be recorded and reviewed by the Chief Investigator. If an $A E$ is considered to be serious (an $S A E$ ), it will be reviewed for causality and expectedness by an independent rater and the sponsor's representative. The Trial Steering Committee (TSC) will be informed of the number, nature and review outcome for all SAEs and will be asked to recommend any necessary actions. SAEs will be reported to the NHS Research Ethics Committee as appropriate.

\section{Dissemination Plans}

Trial findings will be disseminated in scientific publications, including feasibility outcomes and process evaluation findings. Findings will be disseminated to participants' and patient organizations. LEAP members will participate in dissemination including use of social media to disseminate findings, producing leaflets for wide distribution and submitting a summary of findings to a non-academic journal. Findings will be presented at patient events and at local, national and international conferences.

\section{Discussion}

CBTp is an evidence-based psychological therapy recommended for psychosis patients within the UK. However, only a minority of patients are offered CBTp. Limited resources have been cited as a prominent reason for this lack of access, leading to calls for shorter forms of CBTp to be developed that can be delivered by briefly trained therapists.

We have responded to this call by developing a brief and targeted form of CBTp for distressing voices Guided, self-help intervention for voices ('GiVE'). We have generated preliminary evidence for the effectiveness of GiVE when delivered by highly trained therapist (Clinical Psychologists). The next step is to evaluate the experience of stakeholders when GiVE is delivered by briefly trained therapists (Assistant Psychologists). The current study will explore the feasibility of conducting this evaluation in the form of a pragmatic three-arm parallel group superiority 1:1:1 RCT comparing GiVE to an active control intervention (Supportive Counselling) and Treatment as Usual.

CBTp offered in these less resource-intensive forms has the potential to generate benefits for: 1) individual patients (reduced distress and enhanced recovery and enhanced quality of life); 2) service-level patient benefit (increased access to evidence-based psychological therapies); and 3) economic benefits to the NHS (in terms of the reduced use of mental health inpatient services).

\section{Trial Status}


Recruitment to the trial commenced in February 2019 (study protocol - version 1, dated 05/11/18). At present, recruitment and data collection will continue until July 2020.

\section{Abbreviations}

AP - Assistant Psychologists

CBTp - Cognitive Behaviour Therapy for psychosis

CC - Care Co-ordinator

CHOICE - CHoice of Outcome In Cbt for psychoses

CSRI - Client Service Receipt Inventory

DMEC - Data Monitoring \& Ethics Committee

DSM - Diagnostic and Statistical Manual for Mental Disorders

GiVE - Guided self-help CBT intervention for voices

HPSVQ - Hamilton Program for Schizophrenic Voices Questionnaire

HRQoL - Health related quality of life

MCID - Minimum clinically important difference

MRC - Medical Research Council

NICE - National Institute for Health and Care Excellence

NHS - National Health Service

NIHR - National Institute for Health Research

PIS - Participant Information Sheet

QALY - Quality-Adjusted Life Years

PSYRATS - Psychotic Symptoms Rating Scale

RA - Research Assistant

RCT - Randomised Controlled Trial

SC - Supportive Counselling 
SCID-5 - Structured Clinical Interview for DSM-5 disorders

SF12 - Short Form 12

TAU - Treatment As Usual

TOPF-UK - Test of Premorbid Functioning - UK

TSC - Trial Steering Committee

\section{Declarations}

\section{Ethics approval and Consent to Participate}

The study is sponsored by Sussex Partnership NHS Foundation Trust. NHS Research Ethics Committee (London - Surrey - reference number 18/LO/ 2091), Health Research Authority and local Research Governance approval was granted before the commencement of the trial (study protocol - version 1 , dated 05/11/18 [see Table 1 for approved amendments to protocol]). Participants will provide written informed consent prior to the completion of any study procedures.

\section{Acknowledgements}

We would like to acknowledge the significant contribution to the study that is being made by the APs (Emogen Campbell, Sophie Green, Debbie Sheffield, and Sasha Stone) and Research Assistants (Maisha Murshed and Suzanne Neumann). We also appreciate the advice and assistance that is being offered by Becky Whitfield, Peter Garwood, Jake Ellwood, Tina Perry and our Lived Experience Consultants.

\section{Authors' contributions $\{31 \mathrm{~b}\}$}

$\mathrm{MH}$ is the $\mathrm{Cl}$ of the study and accountable for all aspects of the work in ensuring that questions related to the accuracy or integrity of any part of the work are appropriately investigated and resolved. $\mathrm{MH}, \mathrm{BC}$ and $\mathrm{CB}$ took responsibility for the main drafting of the manuscript. All authors made substantial contributions to conception and design. All authors have been involved in drafting the manuscript or revising it critically for important intellectual content. All authors read and approved the final manuscript.

\section{Funding $\{4\}$}

This project is funded by the Research for Patient Benefit programme, a National Institute for Health Research grant (ref. number PB-PG-0317-20029). The funding body has not influenced the design, conduct, analysis or dissemination of this study. The views expressed in this publication are those of the author(s) and not necessarily those of the NHS, NIHR or the Department of Health.

\section{Availability of data and materials $\{29\}$}


Not currently applicable. The fully anonymised datasets generated and/or analysed (and associated statistical code) during the current study will be available from the corresponding author on reasonable request following the publication of results.

\section{Ethics approval and consent to participate $\{24\}$}

The study is sponsored by Sussex Partnership NHS Foundation Trust who will provide at least annual monitoring and audit of the trial and governance procedures. NHS Research Ethics Committee (London Surrey, reference number 18/LO/ 2091), Health Research Authority and local Research Governance (at both sites) approval were granted before the commencement of the trial.

\section{Competing interests $\{28\}$}

There is a potential conflict of interest as some members of the research team $(\mathrm{MH}, \mathrm{CS} \& \mathrm{CH})$ are also authors on the self-help book and/or published workbook being used within the GiVE intervention. Therefore, the research team agrees to publish the results of this feasibility trial, irrespective of the findings.

\section{Author details}

Mark Hayward (corresponding author)

School of Psychology, University of Sussex, Brighton BN1 9RH \& Research \& Development Department, Sussex Partnership NHS Foundation Trust, Hove, BN3 7HZ

m.i.hayward@sussex.ac.uk

Clio Berry

School of Psychology, University of Sussex, Brighton BN1 9RH

c.berry@sussex.ac.uk

Ben Cameron

Research \& Development Department, Pennine Care NHS Foundation Trust, 225 Old St, Ashton-underLyne OL6 7SR

Ben.cameron@nhs.net

Kate Arnold

Research \& Development Department, Sussex Partnership NHS Foundation Trust, Hove, BN3 7HZ Kate.arnold2@sussexpartnership.nhs.uk 
Katherine Berry

Faculty of Biology, Medicine \& Health, University of Manchester, M13 9PL

Katherine.berry@manchester.ac.uk

Stephen Bremner

Brighton \& Sussex Medical School, University of Sussex, Brighton BN1 9RH

s.bremner@sussex.ac.uk

Kate Cavanagh

School of Psychology, University of Sussex, Brighton BN1 9RH

k.cavanagh@sussex.ac.uk

David Fowler

School of Psychology, University of Sussex, Brighton BN1 9RH

D.Fowler@sussex.ac.uk

Heather Gage

School of Biosciences \& Medicine, University of Surrey GU27XH

h.gage@surrey.ac.uk

Kathryn Greenwood

School of Psychology, University of Sussex, Brighton BN1 9RH

k.e.greenwood@sussex.ac.uk

Cassie Hazell

Social Sciences Department, University of Westminster, London W1W 6UW

c.hazell@westminster.ac.uk

Anna-Marie Jones

Research \& Development Department, Sussex Partnership NHS Foundation Trust, Hove, BN3 7HZ Anna-marie.jones@sussexpartnership.nhs.uk 
Sam Robertson

Research \& Development Department, Sussex Partnership NHS Foundation Trust, Hove, BN3 7HZ Sam.robertson@sussexpartnership.nhs.uk

Clara Strauss

University of Sussex \& Sussex Partnership NHS Foundation Trust, BN3 7HZ c.y.strauss@sussex.ac.uk

\section{References}

1. van der Gaag M, Valmaggia LR, Smit $F$. The effects of individually tailored formulation- based cognitive behavioural therapy in auditory hallucinations and delusions: A meta-analysis. Schizophr Res. 2014: 156:30-37

2. Jones C, Cormac I, Silveira da Mota Neto JI, Campbell C. Cognitive behaviour therapy for schizophrenia. Cochrane Database Systematic Review. 2004 (4): CD000524.

3. Wykes T, Steel C, Everitt B, Tarrier N. Cognitive Behavior Therapy for Schizophrenia: Effect Sizes, Clinical Models, and Methodological Rigor. Schizophrenia Bull. 2008: 34;523-537.

4. National Institute for Health and Care Excellence. Psychosis and schizophrenia in adults: Treatment and management (CG178). 2014. London: National Institute for Health and Care Excellence. Available at http://guidance.nice.org.uk/CG178.

5. Haddock G, Eisner E, Boone C, Davies G, Coogan C, Barrowclough C. An investigation of the implementation of NICE-recommended CBT interventions for people with schizophrenia. J Ment Health. 2014: 23;162-165.

6. The Royal College of Psychiatrists. National Clinical Audit of Psychosis - National Report for the Core Audit. 2018. London: Healthcare Quality Improvement Partnership.

7. Ince P, Haddock G, Tai S. A systematic review of the implementation of recommended psychological interventions for schizophrenia: Rates, barriers, and improvement strategies. Psychol Psychother-T. 2016: 89;324-350.

8. NHS England. Five year forward view. 2014. https://www.england.nhs.uk/wp-content/ uploads/2014/10/5yfv-web.pdf. Accessed 5 Oct 2019.

9. NHS. The NHS long term plan. 2019. https://www.longtermplan.nhs.uk/. Accessed 5 Oct 2019

10. Hazell C, Hayward M, Cavanagh K. Strauss C. A systematic review and meta-analysis of low intensity CBT for psychosis. Clin Psychol Rev. 2016: 45;183-192. 
11. Birchwood M. Trower P. The future of cognitive-behavioural therapy for psychosis: not a quasineuroleptic. Brit J Psychiat. 2006: 188;107-108.

12. Freeman D, Dunn G, Startup H, Pugh K, Cordwekk J, Mander H., et al. Effects of cognitive behaviour therapy for worry on persecutory delusions in patients with psychosis (WIT): a parallel, single-blind, randomised controlled trial with a mediation analysis. Lancet Psychiat. 2015: 380;305-313.

13. Waller H, Emsley R, Freeman D, Bebbington P, Dunn R, Fowler D. et al. Thinking Well: A randomised controlled feasibility study of a new CBT therapy targeting reasoning biases in people with distressing persecutory delusional beliefs. J Behav Ther Exp Psy. 2015: 48;82-89.

14. Craig TKJ, Rus-Calafell M, Ward T, Leff JP, Huckvale M, Howarth E. et al. AVATAR Therapy for auditory verbal hallucinations in psychosis patients: a single-blind randomized controlled trial. Lancet Psychiat. 2018: 5;31-40.

15. van der Gaag M, Van Oosterhout B, Daalman K, Sommer IE Korrelboom K. Initial evaluation of the effects of competitive memory training (COMET) on depression in schizophrenia-spectrum patients with persistent auditory verbal hallucinations: a randomized controlled trial. Brit J Clin Psychol. 2012: $5 ; 158-171$

16. Turkington D, Kingdon D, Turner T. Effectiveness of a brief cognitive- behavioural therapy intervention in the treatment of schizophrenia. Brit J Psychiat. 2002: 180;523-7.

17. Turkington D, Kingdon D, Rathod S, Hammond K, Pelton J, Mehta R. Outcomes of an effectiveness trial of cognitive-behavioural intervention by mental health nurses in schizophrenia. Brit J Psychiat. 2006: $189 ; 36-40$.

18. Harding H, Landau S, Fornells-Ambrojo M, Jolley S, McCrone P, Halkoree R. et al. Improving implementation of evidence-based practice for people with psychosis through training the wider workforce: Results of the GOALS randomized controlled trial. J Behav Ther Exp Psy. 2018. 59;121128.

19. Jolley S, Onwumere J, Kuipers E, Craig T, Moriarty A, Garety, P. Increasing access to psychological therapies for people with psychosis: predictors of successful training. Behav Res Ther. 2013: 50;457462 .

20. Richards DA, Ehlers D, McMillan D, Taylor R, Byford S, Warren F. et Cost and outcome of behavioural activation versus cognitive behaviour therapy for depression (COBRA): a randomised, controlled, noninferiority trial. Lancet. 2016: 388;871-880.

21. Hayward M, Strauss C, Kingdon D. Overcoming Distressing Voices $\left(2^{\text {nd }}\right.$ Ed). 2018. Constable \& Robinson: London. 
22. Hazell CM, Hayward M, Cavanagh K, Jones A.-M, Strauss C. Guided self-help cognitive behavioral intervention for VoicEs (GiVE): study protocol for a pilot randomized controlled trial. Trials. 2016: $17 ; 351$

23. Hazell C, Hayward M, Cavanagh K, Jones A-M, Strauss C. Guided self-help cognitive-behaviour Intervention for VoicEs (GiVE): Results from a pilot randomised controlled trial. Schizophr Res. 2017: 195;441-447.

25. Van Lieshout RJ, Goldberg JO. Quantifying self-reports of auditory verbal hallucinations in person with psychosis. Can J Beh Sci. 2007: 39;73-7

26. First MB, Williams JBW, Karg RS, Spitser RL. Structured clinical interview for DSM-5 disorders (SCID5). 2016. Washington, DC: American Psychiatric Association.

27. Wechsler D. The Test of Premorbid Functioning (TOPF). 2011. San Antonio TX: The Psychological Corporation.

28. Hazell C, Hayward M, Strauss C. Kingdon D. An introduction to self-help for distressing voices. 2018. London: Robinson.

29. AVATAR Study. Manual for supportive counseling. Unpublished therapy protocol. 2014. Kings College London.

30. Haddock G, Devane S, Bradshaw T, McGovern J, Tarrier N, Kinderman P, et al. An investigation into the psychometric properties of the Cognitive Therapy Scale for Psychosis (CTS-Psy). Behav Cogn Psychoth. 2001: 29;221-234.

31. Thornicroft G, Becker T, Knapp M, Knudsen HC, Schene A, Tansella, M. CSRI European version. International outcome measures in mental health (Chapter 9). 2006. Royal College of Psychiatrists: Trowbridge, Wiltshire.

32. Whitehead AL, Julious SA, Cooper CL, Campbell MJ. Estimating a sample size for a pilot randomised trial to minimise the overall trial sample size for the external pilot and the main trial for continuous outcome variable. Stat Methods Med Res. 2016: 25;1057-1073.

33. Sim J, Lewis M. The size of a pilot study for a clinical trial should be calculated in relation to considerations of precision and efficiency. J Clin Epidemiol. 2012: 65;301-308.

34. Teare MD, Dimairo M, Shepherd N, Hayman A, Whitehead A, Walters SJ. Sample size requirements to estimate key design parameters from external pilot randomised controlled trials: a simulation study. Trials. 2014: 15;264.

35. Haddock G, McCarron J, Tarrier N, Faragher EB. Scales to measure dimensions of hallucinations and delusions: The psychotic symptom rating scales (PSYRATS). Psychol Med. 1999: 29;879-889. 
36. Woodward TS, Jung K, Hwang H, Yin J, Taylor L, Menon M, et al. Symptom dimensions of the psychotic symptom rating scales in psychosis: a multisite study. Schizophr Bull. 2014: 40;265-274.

37. Kim SH, Jung HY, Hwang SS, Chang JS, Kim Y, Ahn YM, Kim YS., 2010. The usefulness of a selfreport questionnaire measuring auditory verbal hallucinations. Prog Neuro-Psychoph 34, 968-973.

38. Zigmund AS, Snaith RP. The hospital anxiety and depression scale. Acta Psychiat Scand. 1983: 67;361-370.

39. 39. Green C, Freeman D, Kuipers E, Bebbington P, Fowler D, Dunn G. et al. Measuring ideas of persecution and reference: the Green et al Paranoid Thought Scales (G-PTS). Psychol Med. 2008: 38;101-11.

40. Fowler D, Freeman D, Smith B, Kuipers E, Bebbington P, Bashworth, H. et al. The Brief Core Schema Scale (BCSS): psychometric properties and associations with paranoia and grandiosity in nonclinical and psychosis samples. Psychol Med. 2006: 36:749-59.

41. Chadwick P, Lees S, Birchwood M. The revised Beliefs About Voices Questionnaire (BAVQ-R). Brit J Psychiat. 2000: 177;229-232.

42. Hayward M, Denney J, Vaughan S, Fowler D. The Voice and You: Development and psychometric evaluation of a measure of relationships with voices. Clin Psychol Psychot. 2008: 15;45-52.

43. Birtchnell J, Hammond S, Horn E, De Jong C, Argyroula K. A cross national comparison of a shorter version of the Person's Relating to Others Questionnaire. Clin Psychol Psychot. 2013: 20;36-48.

44. Greenwood KE, Sweeney A, Williams S, Garety P, Kuipers E, Scott J, et al. CHoice of Outcome In Cbt for psychosEs (CHOICE): the development of a new service user-led outcome measure of CBT for psychosis. Schizophr Bull. 2010:36;126-135.

45. Strauss C, Hayward M, Hazell C. (in preparation). The Voice Impact Scale (VIS): Establishing the psychometric properties of a measure of the impact of hearing voices.

46. Mundt JC, Marks IM, Shear MK, Greist JM. The Work and Social Adjustment Scale: A simple measure of impairment in functioning. Brit J Psychiat. 2002: 180;461-4.

47. Rybarczyk B. Social and Occupational Functioning Assessment Scale (SOFAS). In: Kreutzer J.S., DeLuca J., Caplan B. (eds) Encyclopedia of Clinical Neuropsychology. 2011. Springer: New York.

48. McGuire-Snieckus R, McCabe R, Catty J, Hansson L,Proebe S. A new scale to assess the therapeutic relationship in community mental health care: STAR. Psychol Med. 2007: 37;85-95.

49. Devilly GJ, Borkovec TD. Psychometric properties of the credibility/expectancy questionnaire. J Beh Ther Exp Psy. 2000: 31;73-86. 
50. Brooks, R. EuroQol: The current state of play. Health Policy. 1996: 37;53-72.

51. Ware JE, Kosinki M, Keller SD. SF-12: How to Score the SF-12 Physical and Mental Health Summary Scales. 1995. Boston, MA: The Health Institute, New England Medical Centre.

53. Moore GF, Audrey S, Barker M, Bond L, Bonell C. Hardeman W. et al. Process evaluation of complex interventions: Medical Research Council guidance. Brit Med J. 2015: 350;h1258.

54. Braun V, Clarke V. Using thematic analysis in psychology. Qual Res Psychol. 2006: 3;77-101.

55. Medical Research Council (UK). (1998). Guidelines for Good Clinical Practice in Clinical Trials. 1998. London, England: MRC.

\section{Table}

Table 1 Protocol changes

\begin{tabular}{|c|c|}
\hline $\begin{array}{l}\text { Protocol } \\
\text { version }\end{array}$ & Details of change \\
\hline \multirow[t]{3}{*}{$\begin{array}{l}\text { Version } 2 \\
(02 / 01 / 2019)\end{array}$} & $\begin{array}{l}\text { Addition of exclusion criterion for patients currently detained in hospital under a } \\
\text { section of the Mental Health Act. }\end{array}$ \\
\hline & $\begin{array}{l}\text { Addition of Test of Premorbid Functioning as relevant to eligibility assessment of } \\
\text { presence of serious learning disability. }\end{array}$ \\
\hline & Addition of statement of intent to audio-record participant assessments. \\
\hline \multirow{2}{*}{$\begin{array}{l}\text { Version } 3 \\
(17 / 04 / 2019)\end{array}$} & Reduction of lower age threshold for inclusion from 18 years to 16 years. \\
\hline & Removal of statement of intent to audio-record participant assessments. \\
\hline \multirow[t]{2}{*}{$\begin{array}{l}\text { Version } 4 \\
(14 / 08 / 2019)\end{array}$} & $\begin{array}{l}\text { Addition of questionnaire for referring and non-referring clinicians to explore their } \\
\text { decision-making around referring patients with psychosis to CBT generally and the } \\
\text { GiVE intervention specifically as part of process evaluation. }\end{array}$ \\
\hline & $\begin{array}{l}\text { Addition of qualitative interview with a subsample of therapy participants to explore } \\
\text { their experiences of therapy participation, outcomes, and potential mechanisms of } \\
\text { change as part of process evaluation. }\end{array}$ \\
\hline $\begin{array}{l}\text { Version } 5 \\
(25 / 09 / 2019)\end{array}$ & Typographical correction regarding list of participant assessments. \\
\hline
\end{tabular}

\section{Figures}




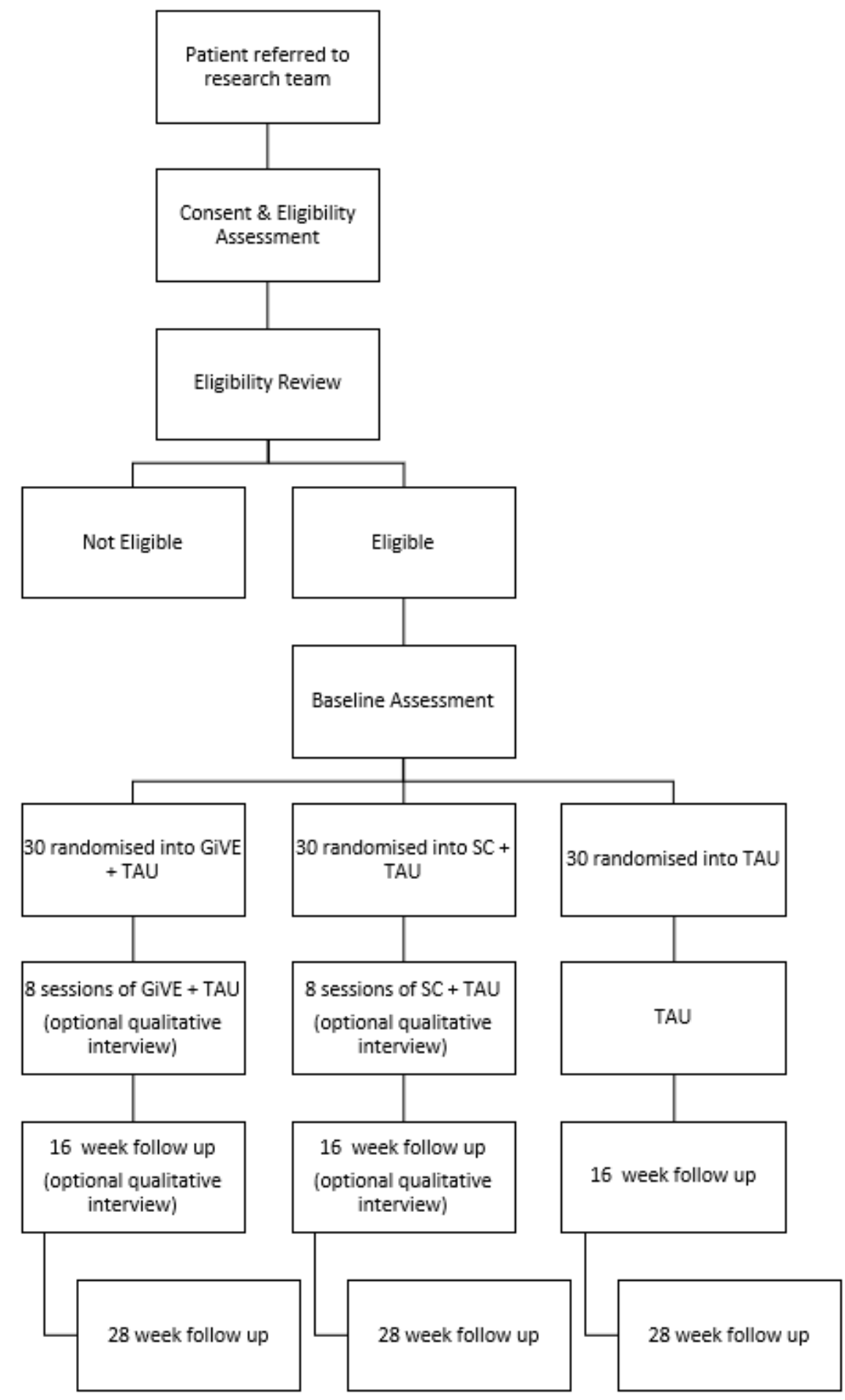

Figure 1

Trial/recruitment flowchart 


\begin{tabular}{|c|c|c|c|c|c|c|}
\hline \multirow{2}{*}{ TIME POINT } & \multirow{2}{*}{$\begin{array}{l}\text { Screening } \\
-t 1\end{array}$} & \multirow{2}{*}{$\begin{array}{c}\text { Baseline } \\
-t 2\end{array}$} & \multirow{2}{*}{$\begin{array}{c}\text { Allocation } \\
0 \\
0\end{array}$} & \multirow{2}{*}{$\begin{array}{c}\text { Intervention } \\
\text { (weeks) } \\
8\end{array}$} & \multicolumn{2}{|c|}{$\begin{array}{l}\text { Follow up } \\
\text { (weeks) }\end{array}$} \\
\hline & & & & & 16 & 28 \\
\hline \multicolumn{7}{|l|}{ ENROLMENT: } \\
\hline Informed consent & $\mathbf{X}$ & & & & & \\
\hline & & & & & & \\
\hline Participant Details & $\mathrm{X}$ & & & & & \\
\hline $\begin{array}{l}\text { Information About You } \\
\text { (demographics) }\end{array}$ & $\mathbf{x}$ & & & & & \\
\hline $\begin{array}{l}\text { Hamilton Program for } \\
\text { Schizophrenia Voices } \\
\text { Questionnaire }\end{array}$ & $\mathbf{x}$ & & & & $\mathbf{X}$ & $\mathbf{x}$ \\
\hline SCID & $\mathbf{X}$ & & & & & \\
\hline TOPF-UK & $\mathrm{X}$ & & & & & \\
\hline RANDOMISATION: & & & $\mathbf{X}$ & & & \\
\hline \multicolumn{7}{|l|}{ INTERVENTIONS: } \\
\hline \multicolumn{7}{|l|}{ GiVE } \\
\hline \multicolumn{7}{|l|}{ SC } \\
\hline \multicolumn{7}{|l|}{ TAU } \\
\hline \multicolumn{7}{|l|}{ ASSESSMENTS: } \\
\hline & & & & & & \\
\hline Voice Impact Scale & & $\mathbf{X}$ & & & $\mathbf{X}$ & $\mathbf{x}$ \\
\hline $\begin{array}{l}\text { Psychotic Symptoms Rating } \\
\text { Scale - Auditory } \\
\text { Hallucinations }\end{array}$ & & $\mathrm{x}$ & & & $\mathbf{X}$ & $\mathrm{x}$ \\
\hline $\begin{array}{l}\text { The Hospital Anxiety and } \\
\text { Depression Scale }\end{array}$ & & $\bar{x}$ & & & $\mathbf{X}$ & $\mathbf{x}$ \\
\hline $\begin{array}{l}\text { Choice of Outcome In Cbt } \\
\text { for psychosES }\end{array}$ & & $\bar{x}$ & & & $\bar{X}$ & $\bar{x}$ \\
\hline $\begin{array}{l}\text { The Brief Core Schema } \\
\text { Scale }\end{array}$ & & $\mathbf{x}$ & & & $\mathbf{X}$ & $\mathbf{x}$ \\
\hline Voice and You & & $\mathbf{X}$ & & & $\mathbf{X}$ & $\mathbf{X}$ \\
\hline $\begin{array}{l}\text { Beliefs about voices } \\
\text { questionnaire }\end{array}$ & & $\mathrm{X}$ & & & $\mathrm{X}$ & $\bar{x}$ \\
\hline $\begin{array}{l}\text { Persons relating to others } \\
\text { questionnaire }\end{array}$ & & $\mathbf{X}$ & & & $\mathbf{X}$ & $\mathbf{x}$ \\
\hline Paranoid Thoughts Scale & & $\mathbf{X}$ & & & $\mathbf{X}$ & $\mathbf{X}$ \\
\hline $\begin{array}{l}\text { Therapy Credibility and } \\
\text { Expectancy Questionnaire }\end{array}$ & & & & $\mathrm{X}$ & & \\
\hline $\begin{array}{l}\text { Scale to assess therapeutic } \\
\text { relationships (therapist) }\end{array}$ & & & & & $\mathbf{X}$ & \\
\hline $\begin{array}{l}\text { Scale to assess therapeutic } \\
\text { relationships (patient) }\end{array}$ & & & & & $\mathbf{X}$ & \\
\hline $\begin{array}{l}\text { Client Service Receipt } \\
\text { Inventory-UK }\end{array}$ & & $\mathbf{x}$ & & & $\mathbf{X}$ & $\mathbf{x}$ \\
\hline EQ5D-5L & & $\mathbf{X}$ & & & $\bar{X}$ & $\mathbf{x}$ \\
\hline SF-12 & & $\mathrm{X}$ & & & $\mathrm{X}$ & $x$ \\
\hline $\begin{array}{l}\text { Work and Social Adjustment } \\
\text { Scale }\end{array}$ & & $\mathrm{X}$ & & & $\mathrm{X}$ & $\mathrm{x}$ \\
\hline $\begin{array}{l}\text { Social and Occupational } \\
\text { Functioning Scale }\end{array}$ & & $\mathbf{X}$ & & & $\mathbf{X}$ & $\mathbf{x}$ \\
\hline $\begin{array}{l}\text { Qualitative interview } \\
\text { (optional for GiVE and SC } \\
\text { participants) }\end{array}$ & & & & $\mathbf{x}$ & $\mathrm{x}$ & \\
\hline
\end{tabular}

\section{Figure 2}

\section{SPIRIT figure}

\section{Supplementary Files}

This is a list of supplementary files associated with this preprint. Click to download. 
- SPIRITChecklist.doc

- RECfavourableopinion.pdf

- Awardletter.pdf

- Appendix1.docx

- Appendix2.docx 